\title{
ULTRASOUND DIAGNOSIS OF ACHILLES TENDON PATHOLOGY IN RUNNERS
}

\author{
N. MAFFULLI, MD*, R. REGINE, MD, M. ANGELILLO, MD, G. CAPASSO, MD* and S. FILICE, MD
}

Second Medical School, First Institute of Radiology and *First Medical School, First Institute of Orthopaedics and Traumatology, University of Naples, Italy

\section{ABSTRACT}

The great upsurge in popularity of running activities has increased the number of athletes presenting with pathology of the Achilles tendon.

A clinical and ultrasonic study was performed on 47 middle and long distance runners referred to the Authors with such problems.

The results of this study can be grouped as follows:

1. paratendonitis: enlargement of the antero-posterior diameter of the tendon, and hyperechogenicity of Kager's triangle;

2. tendonitis (with or without paratendonitis): thickening of the tendon, with the presence of degenerative nodules;

3. enthesopathy: thickening of the distal part of the tendon, enlargement of the hypoechogenic area behind the tendon itself and microcalcification.

Key words: Ultrasound, Achilles tendon, Distance running

\section{INTRODUCTION}

Achilles tendinopathy is a recognised complication of running activities. Aetiological factors include running surfaces, poor flexibility and strength, partially worn out shoes, training errors and lower limb biomechanical factors (Taunton, 1986).

Until recently the diagnosis of this condition was based almost entirely on clinical examination, as radiology was technically unsuitable (Vazelle et al, 1981), and xeroradiography too expensive for routine use.

Ultrasound has been used to investigate tendon and muscular structures. For example, Vazelle et al (1982) used both ultrasound and xeroradiography to define the normal appearance of the Achilles tendon and its relationship with the plantaris tendon. Vazelle et al (1981) had also used mammographic equipment to study lesions of the Achilles tendon. More recently, Laine et al (1984) used ultrasound to assess the presence of Achilles tendon rupture and paratendonitis, stressing the importance of ultrasound in the follow up of the disease.

We have used ultrasound not only to confirm the diagnosis, but also to identify the type of tendon alteration, its anatomo-pathological stage, and to compare our results with previous studies.

\section{CLASSIFICATION}

From the clinical point of view, various pathological forms of Achilles tendon pathology are recognised (Puddu et al, 1976):

1. tenalgia: painful tendon, without evidence of alteration in the tendon itself;

2. paratendonitis: the inflammatory processes involve the highly vascularised peritendinous connective tissue;

3. tendonitis (pure, or associated with paratendonitis): there is a pathological involvement of the tendon itself, either as a whole or in a well localised portion.

\footnotetext{
Address for correspondence:

N. Maffulli, MD

Institute of Child Health

Sports Medicine Laboratory

30 Guilford Street

London WC1
}

These areas are relatively poorly vascularised and can show degenerative phenomena, leading, if not diagnosed, to subcutaneous rupture (Kvist and Kvist, 1980);

4. enthesopathy: where the tendon is inserted into the bone, there are particular features due to the presence of a fibrocartilagineous transitional area with vessels coming from both bone and periostium (Perugia et al, 1981) and one or more bursae. These structures can be involved in inflammatory or degenerative processes.

\section{PATIENTS AND METHODS}

The series presented consists of 47 athletes, all of them middle or long distance runners, training for at least six days per week and for at least one year before the study. They had all been complaining of Achilles tendon problems for an average of $2.5 \pm 6.3$ months before the study. An Orthopaedic surgeon diagnosed Achilles tendon pathology based on clinical examination in all 47 patients.

Ultrasound scan was performed immediately after a radiologist had examined the patients. The ultrasound scan analysis was done on photographs by two radiologists. One of the radiologists had no prior knowledge of the patients. All ultrasonic examinations were performed using a real time ultrasound machine, equipped with a linear $7 \mathrm{MHz}$ probe, and a sectorial $5 \mathrm{MHz}$ probe.

\section{RESULTS}

The results of clinical and ultrasonic examination are shown in Table I.

TABLEI

Clinical and ultrasound diagnosis of alterations of the Achilles tendon in 47 middle and long distance runners.

Clinical examination Ultrasound scan

\begin{tabular}{lcc}
\hline Tenalgia & 12 & 8 \\
Paratendonitis & 18 & 5 \\
Tendonitis & 13 & 11 \\
Tendonitis with paratendonitis & - & 17 \\
Enthesopathy & 12 & 14 \\
Total & 55 & 55 \\
\hline
\end{tabular}

Eight athletes were suffering from bilateral tendon pathology. 
From the analysis of the ultrasound scan photographs, the following normal and pathological features were identified:

1. normal Achilles tendon: 8 athletes were suffering from bilateral tendon pathology. In 39 cases, the normal tendon appeared as a ribbon-like, hypoechogenic structure, delineated by two thin and regular echogenic bands (Fig. 1). Its average thickness was $5.1 \pm 0.4 \mathrm{~mm}$. The thickness of the hypoechogenic portion decreases at

To the right of each photograph there is a corresponding diagram. In the photographs, the " $x$ " and " + " are the markings at which measurements were taken.

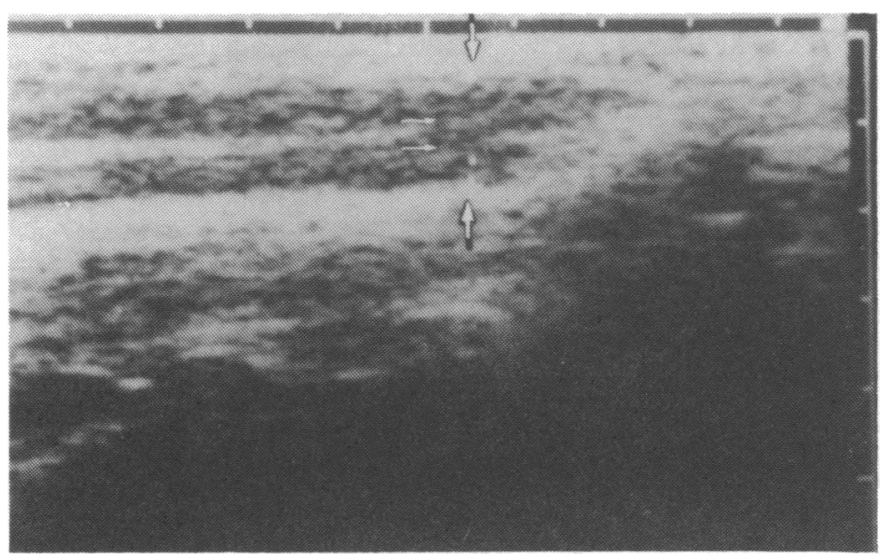

the calcaneal insertion, together with the total tendon thickness. In this area, it was often possible to visualise two hypoechogenic areas, the largest in front of the tendon, above the bony surface, with defined borders. This is the deep bursa (Fig. 2). The second hypoechogenic area is better defined, and is the retrocalcaneal portion of the insertion bursa. Kager's triangle gives a homogeneous ultrasonic image, with increased echogenicity (Fig. 3).

In the diagrams:

BU: bursa; CA: calcaneus; FHL: flexor hallucis longus; $K$ : Kager's triangle TE: Achilles tendon; TI: tibia.

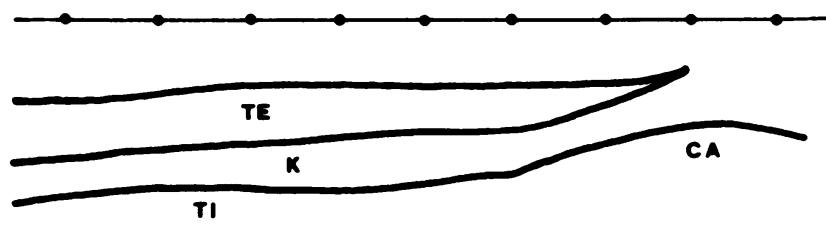

Fig. 1: Ultrasound image of a normal Achilles tendon. The two bigger arrows are placed in the two regular echogenic bands delineating the hypoechogenic structure of the tendon.

The smaller arrows, in the substance of the tendon, show the waveform structure of the tendon itself.
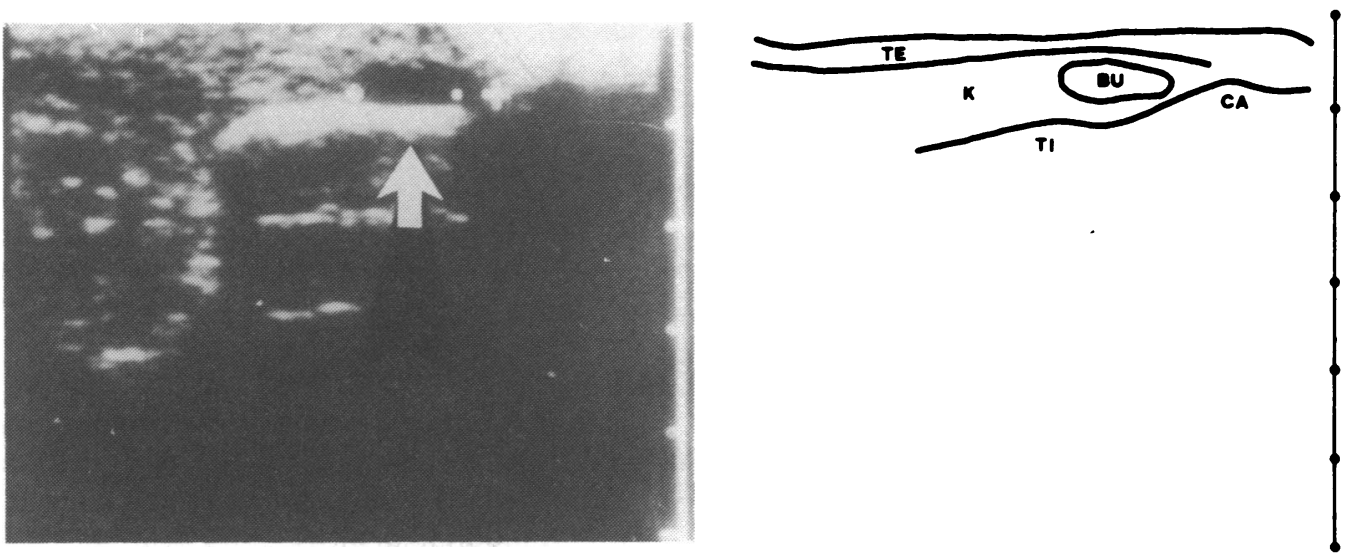

Fig. 2: Ultrasound appearance of a normal pretendinous bursa: hypoechogenic area, in front of the tendon, with well defined borders. The arrow points to the posterior wall of the tendon.
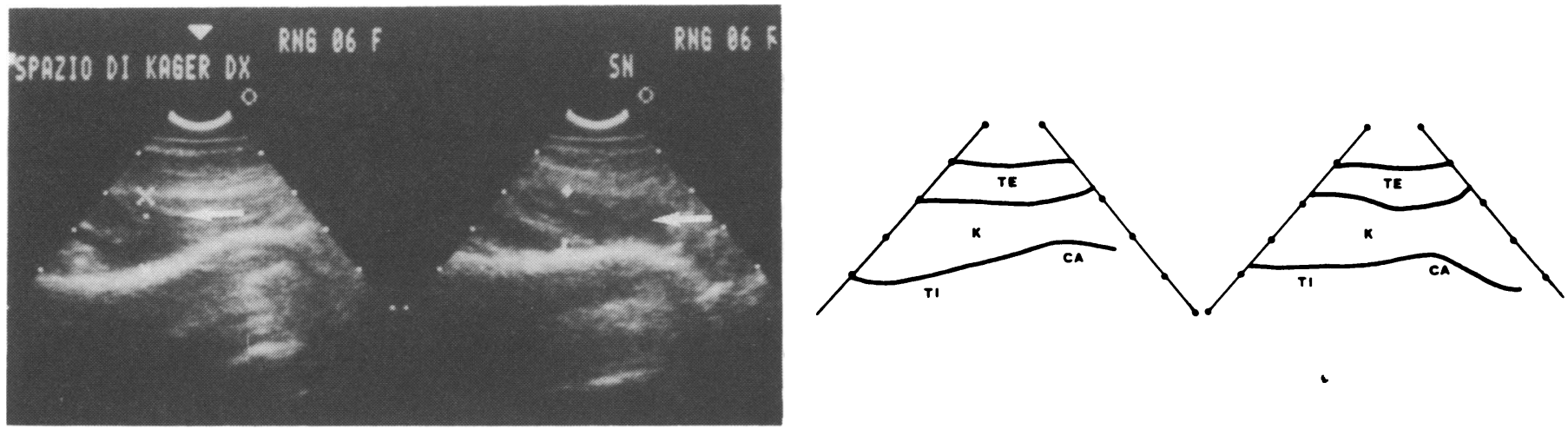

Fig. 3: Kager's triangle: a homogenous triangle, with a high number of echoes. The photograph shows a normal Kager's triangle on both right and left side in a patient suffering from right tenalgia. The " $x$ " (left side) and the "+" (right side) are the measuring points of the width of the triangle. 
In a small but significant number of subjects (eight) with the clinical presentation of tenalgia, the ultrasonic appearance was the same as the normal contralateral tendon.

These patients were examined at a very early stage, so it is assumed that definite anatomo-pathological changes had not yet taken place;

2. paratendonitis: in five cases $(10.7 \%)$, a thickening of the anterior border of the tendon could be demonstrated, together with, in some cases, hyperechogenicity of Kager's triangle. These findings were accompanied by an enlargement of the antero-posterior diameter of the tendon (from $50 \%$ to $100 \%$ as compared with the normal contralateral one, with an average of $78 \pm 14 \%)$, but it was still possible to identify its normal waveform structure (Fig. 4), which was lost in cases of tendonitis;

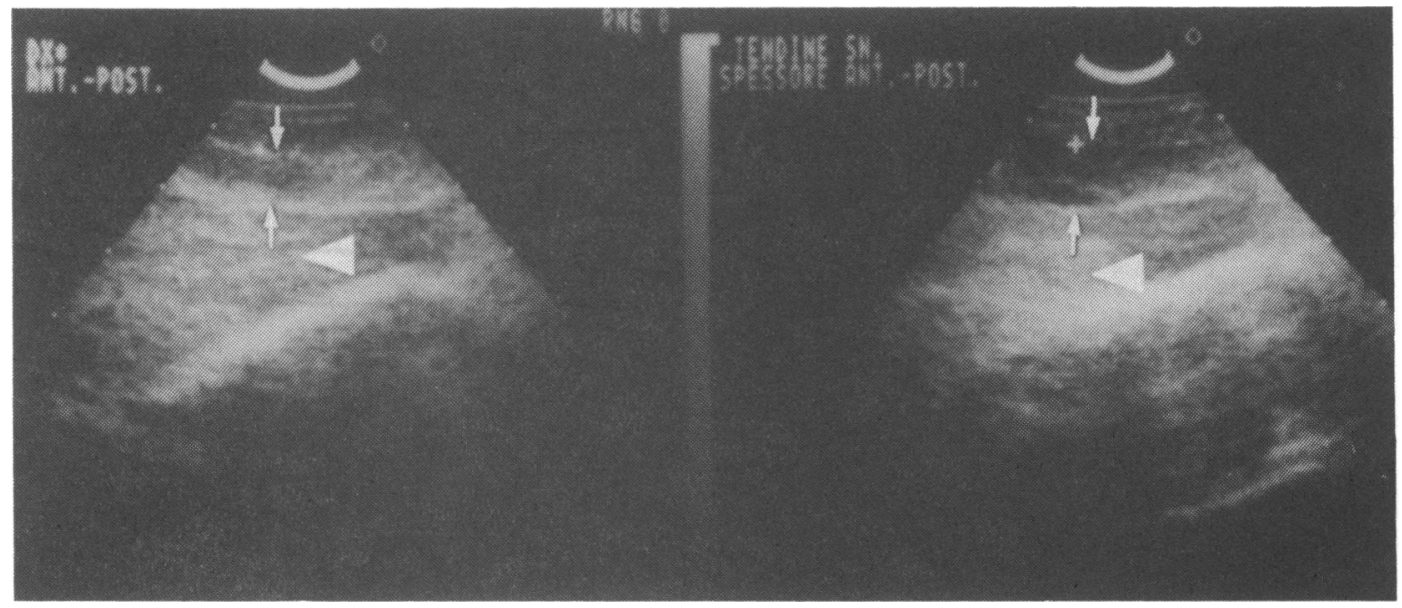

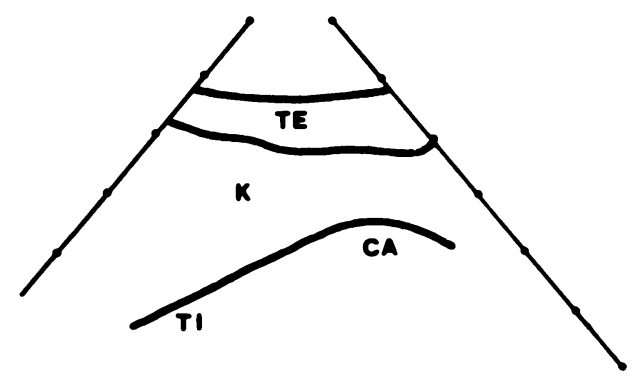

Fig. 4: Paratendonitis (on the right side): when compared to the normal contralateral tendon (on the left), there is an enlargement of the anteroposterior diameter (arrows), with some thickening of the anterior border of

3. tendonitis: a total of 28 cases $(59.6 \%)$ showed ultrasonic features of tendonitis. Two categories were distinguished:

A. pure tendonitis: in seventeen cases $(36.2 \%)$, there was a widenina of the tendon, either partially or

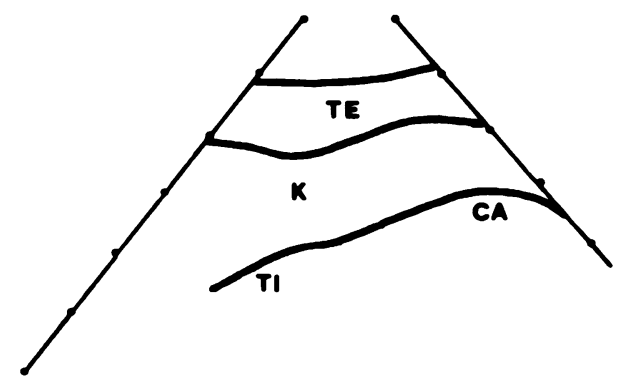

the tendon. The arrows show the anterior and posterior borders of the tendon. The " + " on the right side points to the posterior border of the tendon. The Kager's triangle is not involved in the disease.

throughout its entire length (Fig. 5).

B. tendonitis associated with paratendonitis: in eleven cases $(23.4 \%)$, there was hyperechogenicity of Kager's triangle and of the anterior border of the tendon (Fig. 6).
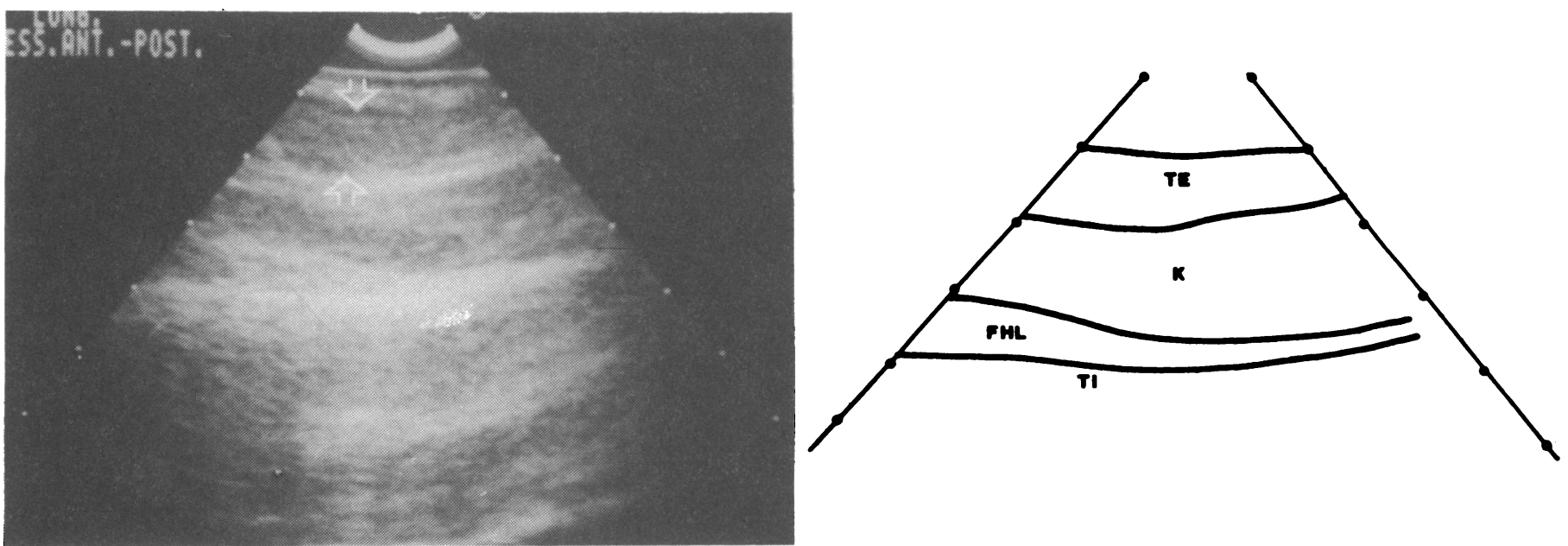

Fig. 5: Tendonitis: the tendon is widened, either for its entire length or partially. The arrows show the point of discrete changes of echogenic pattern. 

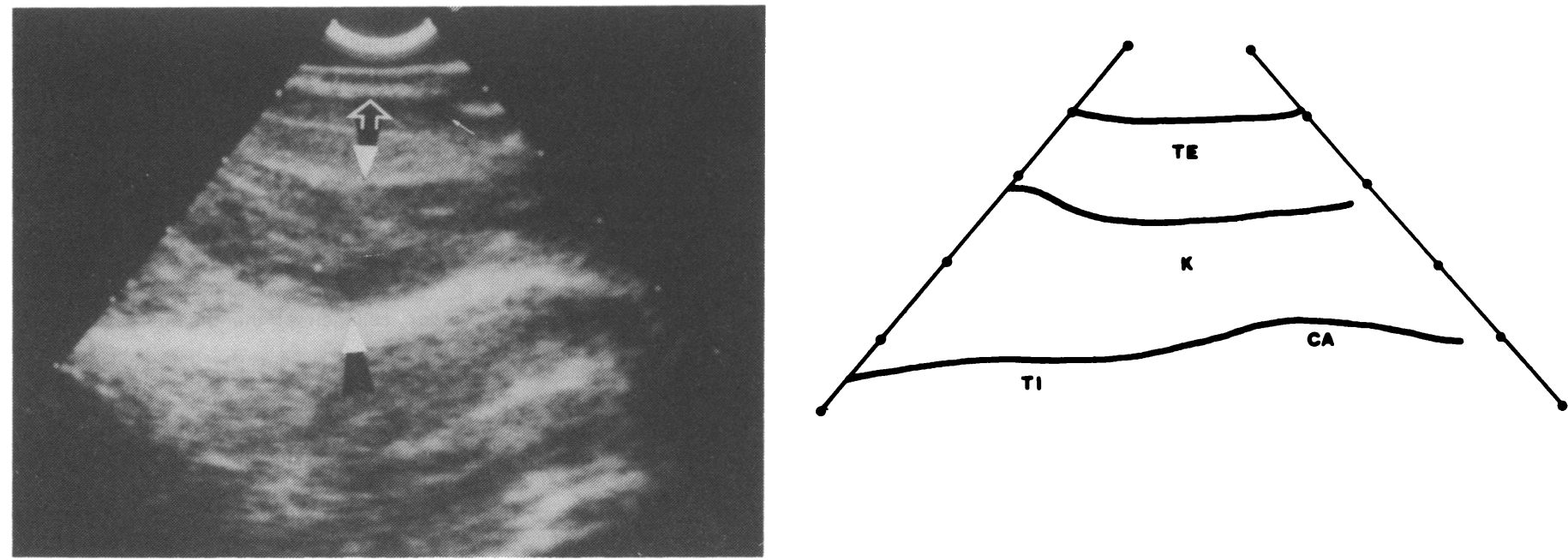

Fig. 6: Tendonitis with paratendonitis: increase in the echogenicity of Kager's triangle (delineated by two triangles) and of the posterior border of the tendon (empty arrows).

The small arrow points to a definite intratendinous area of dishomogeneity.

4. enthesopathy: ultrasound demonstrated and located various alterations in the tendon appearance. In six cases $(12.8 \%)$, there was an enlargement of the hypoechogenic area behind the tendon. In a further eight cases $(17 \%)$, the distal part of the tendon was enlarged, and its echogenicity altered (Fig. 7). Furthermore, plain X-ray films showed some irregularities of the calcaneal profile at the insertion point of the tendon, and the presence of microcalcification.
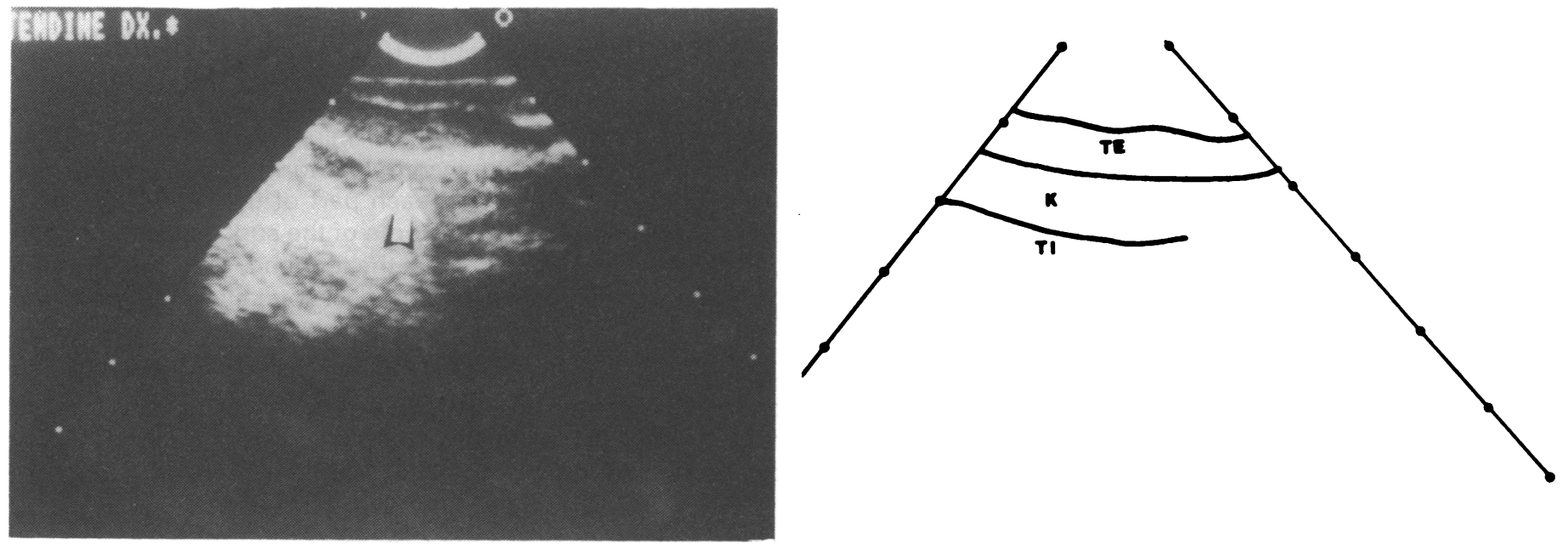

Fig. 7: Enthesopathy: enlargement of the distal part of the tendon, with alterations of the normal pattern of echogenicity. The arrow points to the anterior border of the tendon.

\section{DISCUSSION}

Our observations are in close agreement with those, obtained by other authors, regarding the normal Achilles tendon (Laine et al, 1984; Vazelle et al, 1982). We were able to show that the Achilles tendon has an internal waveform structure, which could match with the anatomical description of the first order fasicles (Perugia et al, 1981).

In paratendonitis, the hyperechogenicity of Kager's triangle could be due to the involvement of the areolar tissue in the inflammatory process. In tendonitis, the small hypoechogenic nodules correspond to areas of degeneration (Perugia et al, 1981) and are the possible site of initiation of tendon rupture (Kvist and Kvist, 1980).

A biochemical explanation of the ultrasonic characteristics of the nodules could be given considering that the proteoglycans constitute the filaments dispersed among and attached to the unorganised fibrils contained in collagen (Benazzo et al, 1982). In tendons, the proteoglycans bound to the fibrils have dermatansulphate lateral chains (Ruggeri et al, 1984), which make them slightly hydrophilic (Scott and Oxford, 1981).

When the proteoglycans are able to float in the matrix, they can retain water, thus influencing the echogenic characteristics of the tendon.

In conclusion, ultrasound gives an extremely precise picture of the alterations of both the peritendinous soft tissues and of the internal structure of the tendon. With the visualisation of these structures, it is possible to formulate an anatomo-pathological based diagnosis, thus being more accurate than relying only on clinical examination. Although xeroradiography can be of comparable cost, it may administer to the subject high doses of radiation and does not give such a precise picture of the internal structure of the tendon. For these reasons, in our opinion xeroradiography is not suitable to supplement the clinician in clinical diagnosis and for longitudinal studies. 
This study was not planned in a double blind fashion, so it is not possible to ascertain the degree of bias in the interpretation of the ultrasound scan. Ultrasound scanning could be used as a readily available, low cost, high safety and high accuracy method in suspected Achilles tendon injuries and in the immediate and late post-operative periods of surgically treated injuries for follow-up purposes.

\section{References}

Benazzo, F., Reale, E., Ruggeri, A., Marchini, M. and Castelli, C., 1982 "Further observations on the relations of proteoglycans with collagen fibrils". Basic and Appl.His. 26 (suppl.).

Campini, R., Ferrari Ciboldi, F., Benazzi, M., Meissner, M. and Rota, L., 1981 "Xeroradiographie zur Diagnostik patologischer Befunde an den Weichteilen des kniegelenkes". Rontgen-Berichte 10: 274-279.

Kvist, H. and Kvist, M., 1980 "The operative treatment of chronic paratenonitis". J.Bone Joint Surg. 62-B: 353-361.

Laine, H., Harjula and Peltokallio, P., 1984 "Real time sonography and tendon injuries of athletes". 5th Congress of the European Federation of Societies for Ultrasound in Medicine and Biology (Euroson '84),
Strasbourg, May 7-11, 40: 7-12.

Perugia, L., Postacchini, F. and Ippolito, E., 1981 "I tendini. Biologia. Patologia. Clinica" (in Italian). Masson Ed. Publisher. Milan. Chapters 2: Anatomia and Chapter 7: Tendiniti. First edition.

Puddu, G., Ippolito, E. and Postacchini, F., 1976 "A classification of Achilles tendon disease". Am.J.Sports Med. 4: 145-149.

Ruggeri, A. and Benazzo, F., 1984 "Collagen, proteoglycans and elastin interactions" in Ruggeri, A. and Motta, P. M. (eds.): Ultrastructure of connective tissue matrix. Martinus Nijhoff/Dr. Junk Publishers, The Hague, The Netherlands, 113-120. First edition.

Scott, J. E. and Oxford, C. R., 1981 "Dermatansulphate-rich proteoglycan associates with rat tail-tendon collagen at the d-band gap region". Biochem.J. 197: 213-217.

Taunton, J. E., 1986 "Medical care of the athlete (first part)". Olympic Review 226: 462-465.

Vazelle, F., Rochcongen, P., Masse, M. and Ramee, A., 1981 "La pathologie du tendon d'Achille: un emploi inattendu du mammographe". J.Radiol. (France) 62: 299-306.

Vazelle, F., Roland, J. J., Rochcongen, P. and Ramee, A., 1982 "Contribution a l'analyse de l'image radiologique du tendon d'Achille. Etude radio-echoanatomique. Localisation du plantaire grele". J.Radiol. (France) 65: 351-358.

\section{BOOK REVIEW}

Title: JOINT PROTECTION AND REHABILITATION IN RHEUMATIC DISORDERS

Author: M. Brattstrom

Publisher: Wolfe Medical, London 1986

Price: $£ 20256$ pages Index Photographs \& line drawings Paperback

Marete Brattstrom presents a comprehensive thesis on joint care divided into two main sections. Part 1 outlines basic joint anatomy and function accompanied by a very brief outline of the common arthritic disorders. There was, I feel one glaring omission in the failure to mention the reactive arthritides such as Reiter's which, in its incomplete form is really one of the commonest forms of arthritis in young people. Part 2 is the meat of the book covering each part of the skeletal system. Sensible advice is given on how to avoid damage and deformity for weak inflamed joints. Some of the suggestions are open to discussion but in the main the recommendations are presented so logically and clearly that the tendency is to think, "that's obvious, just what I tell my patients". We may indeed tell them a little but unless we cover all aspects of daily living joints will still be prematurely destroyed.

The book is therefore primarily written for occupational therapists although all involved in dealing with arthritis should be aware of the principles. One comment especially provides food for thought for the readers of this journal "In the present fitness orientated society the rheumatic patient feels even more of an outcast". In summary the basic message is that daily living can be a danger to your joints, if you have arthritis, and the answer is not to not do it, but to do it differently.

G. Hopkins

\section{BOOK REVIEW}

Title: DIAGNOSTIC PICTURE TESTS IN RHEUMATOLOGY

Authors: V. Wright and A. R. Harvey

Publisher: Wolfe Medical, London 1987 Price: $€ 6.50 \quad 128$ pages

Rheumatological conditions occasionally present as sports injuries, and I strongly recommend this excellent little book as a fun way of learning and revising rheumatology. It consists of a series of pictures and accompanying questions, each set centred on a particular condition. The answers are at the back of the book for an instant self-appraisal. The pictures include photographs of skin, joints and eyes, X-rays, histological sections, thermograms, bone scans and electrocardiograms. Apart from a few of the X-rays which suffer from being reduced in size, the pictures are of excellent quality and cover an encyclopaedic range of conditions. The questions are searching and include aetiology, complications, differential diagnosis and management, and to complement them the answers are clear, succinct and quite detailed.

Professor Vernon Wright and Dr. A. R. Harvey must have combed the archives to collect such a variety of pictures. They cover the whole rheumatological spectrum including inflammatory diseases of connective tissue such as rheumatoid arthritis, systemic lupus erythematosis and scleroderma; the seronegative arthritides; degenerative and traumatic conditions and biochemical disorders such as gout and alcaptonuria. The book fits easily into a pocket and will prove very useful not only to clinical and postgraduate students but to other doctors wishing to brush up on their rheumatology. 